\title{
Artificial Neural Network Prediction of Normalized Polarity Parameter for Various Solvents with Diverse Chemical Structures
}

\author{
Aziz Habibi-Yangjeh \\ Deparment of Chemistry, Facthy of Science, Lnwersity of Hohaghegh Ardabili, P.O. Bor 179, Ardabil, Iran \\ E-mail: habibivangiehavahoo.com; ahabibialuma.acir \\ Received October 31, 2006
}

\begin{abstract}
Artificial neural networks (ANNs) are successfully developed for the modeling and prediction of normalized polarity parameter $\left(\mathrm{E}_{\mathrm{T}}{ }^{\mathrm{N}}\right)$ of 216 various solvents with diverse chemical structures using a quantitative-stnucture property relationship. ANN with architecture 5-9-1 is generated using five molecular descriptors appearing in the multi-parameter linear regression (MLR) model. The most positive charge of a hydrogen atom $\left(\mathrm{q}^{+}\right)$. total charge in molecule $\left(\mathrm{q}_{\mathrm{t}}\right)$. molecular volume of solvent $\left(\mathrm{V}_{\mathrm{m}}\right)$, dipole moment $(\mu)$ and polarizability term $\left(\pi_{\mathrm{I}}\right)$ are input descriptors and its output is $\mathrm{E}_{\mathrm{T}} \times$. It is found that properly selected and trained neural network with 192 solvents could fairly represent the dependence of normalized polarity parameter on molecular descriptors. For evaluation of the predictive power of the generated ANN. an optimized network is applied for prediction of the $\mathrm{E}_{\mathrm{T}}^{\mathrm{N}}$ values of 24 solvents in the prediction set. which are not used in the optimization procedure. Correlation coefficient (R) and root mean square error (RMSE) of 0.903 and 0.0887 for prediction set by MLR model should be compared with the values of 0.985 and 0.0375 by ANN model. These improvements are due to the fact that the $\mathrm{E}_{\mathrm{T}}$ " of solvents shows non-linear correlations with the molecular descriptors.
\end{abstract}

Key Words : Quantitative-structure property relationship. Norntalized polarity parameter. Artificial neural networks, Theoretical descriptors

\section{Introduction}

The energetic level of molecules may be modified by interactions with surrounding molecules of solvents and it may be difficult to relate chemical properties to molecular structures. ${ }^{3}$ The strong influence of solvent on chemical and physical processes (for example. reaction rates, selectivity. chemical equilibria. position and intensity of spectral absorption bands and liquid chromatographic separations. etc.) has well established. ${ }^{\mathrm{l}-\delta}$ The use of solvatochromic indicators is a suitable method for studing solute-solvent interactions. since the transition energy of the indicators depends on the solvation's sphere composition and properties. 'The solvatochromic parameter for measuring empirically the polarity of solvents. $\mathrm{E}_{\mathrm{T}}(30)$. is calculated from the maxima of absorbance of the betaine dye as a solution in the solvent under investigation at $25^{\circ} \mathrm{C}$ and at a pressure of 0.1

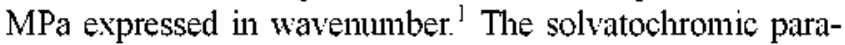
meter is demonstrated to be successful in correlating a wide range of chemical and phỵsical properties involving solutesolvent interactions as well as biological activities of compounds. ' Normalized polarity parameter $\left(\mathrm{E}_{\mathrm{T}}^{\mathrm{N}}\right)$ is a dimensionless "nomalized" scale. defined by equation (1) in reference to tetramethy lsilane (TMS) and water.

$$
E_{T}^{N}=\frac{E_{T}(30)-E_{T}(30)_{T M S}}{E_{T}(30)_{\text {Witer }}-E_{T}(30)_{T M S}}
$$

The macroscopic (bulk) properties of chemical compounds clearly depend on their microscopic (structural) characteristics. Because of importance of solvent effects. it has been of the highest interest to develop quantitative structure properț/activity relationships (QSPR/QSAR). which reflect intermolecular interactions in dense media. Such QSPR/ QSAR correlation equations are usually multi-parametric :-7 $^{-7}$ To obtain a significant correlation. it is crucial that appropriate descriptors be employed. ${ }^{9}$ Famini et al. used theoretical linear solvation energy relationship (TLSER) methodology to correlate $\mathrm{E}_{\mathrm{T}}{ }^{\mathrm{N}}$ of 30 solvents with molecular descriptors. The authors concluded that by the TLSER could predict $E_{T}{ }^{N}$ values for various solvents and provide better understanding of $\mathrm{E}_{\mathrm{T}}{ }^{\mathrm{N}}$ depend on molecular parameters. These descriptors have small cross-correlation. that is to say the descriptors reflect a particular microscopic property nearly without "mixing" or contamination from other descriptors. $10-18$

Table 1 demonstrates the molecular descriptors that have been used in this article. $V_{m}$ is molecular volume of solvent that inversely proportional to the cohesion energy of molecules. The polarizability term $\left(\pi_{1}\right)$ is obtained by dividing the polarizability volume by the molecular volume to produce a unitless. size independent quantity. which indicates the ease with which the electron cloud may be moved or polarized. Dipole moment $(\mu)$ and total charge in molecule $\left(\mathrm{q}_{\mathrm{t}}\right)$ terms demonstrate dipole-dipole interactions. The hydrogen-bond donating ability is divided into two components: $\varepsilon_{\mathrm{A}}$ (the energy difference between the $\varepsilon_{\mathrm{HOMO}}$ of water and $\varepsilon_{\text {LLVO }}$ of solvent) and $\mathrm{q}^{-}$(the most positive charge of a hydrogen atom) of solvent molecule. Analogously. the hydrogen-bond accepting ability is divided into two components: $\varepsilon_{\mathrm{B}}$ (the energy difference between the $\varepsilon_{\text {LLMO }}$ of water and $\varepsilon$ Homo of solvent) and $\mathrm{q}^{-}$(the most negative 
Table 1. The molecular descriptors used in the MLR and ANN models ${ }^{2}$

\begin{tabular}{lllc}
\hline Symbol & Name & Defunition & Units \\
\hline $\mathrm{V}_{\mathrm{m}}$ & Molecular volume & Molecular volume & $\AA^{3}$ \\
$\pi$ & Polarizability index & Polarizability $/ \mathrm{V}_{\mathrm{m}}$ & none \\
$\varepsilon_{\mathrm{A}}$ & Covalent $\mathrm{HB}$ acidity & $0.3-0.01\left(\mathrm{E}_{\mathrm{l}}-\mathrm{E}_{\mathrm{hw}}\right)$ & $\mathrm{heV}$ \\
$\mathrm{q}^{+}$ & Electrostatic HB acidity & Maximum(+) charge on an & $\mathrm{acu}$ \\
& & $\mathrm{H}$ atom & \\
$\mathrm{c}_{\mathrm{B}}$ & Covalent $\mathrm{HB}$ basicity & $0.3-0.01\left(\mathrm{E}_{\mathrm{lw}}-\mathrm{E}_{\mathrm{h}}\right)$ & $\mathrm{heV}$ \\
$\mathrm{q}^{-}$ & Electrostatic HB basicity Maximum(-) charge on ant & $\mathrm{acu}$ \\
& & atom & \\
$\mathrm{q}_{+}$ & Total charge & Total charge on molecule & acu \\
$\mu^{-}$ & Dipole moment & Dipole moment & $\mathrm{D}$ \\
\hline
\end{tabular}

${ }^{a} \mathrm{HeF}=$ hecto-electron folt $\left(\mathrm{I} \mathrm{heV}=100 \mathrm{er}=9.6485 \times 10^{2} \mathrm{~kJ}_{\mathrm{mol}}{ }^{-1}\right)$ acu = atomic charge unit: $\mathrm{D}=$ debye: $\mathrm{HB}=$ hydrogen bond: $\mathrm{E}_{\mathrm{l}}=$ LUMO energy and $\mathrm{E}_{\mathrm{h}}=\mathrm{HOMO}$ energy of the solvent; $\mathrm{E}_{\mathrm{j}}$ and $\mathrm{E}_{\mathrm{h} w}$ refer to the LUMO and HOMO energy for water. respectively.

atomic cliarge) of solvent. ${ }^{11-18}$

Various methods for constructing QSAR/QSPR models have been used including nulti-parameter linear regression (MLR), principal component analysis (PCA) and partial least-squares regression (PLS). In addition. artificial neural networks (ANNs) have beconte popular due to their success where conmplex non-linear relationships exist amongst data. ${ }^{19 . \curvearrowright 1}$ ANNs are biologically inspired computer programs designed to simulate the way in which the human brain processes information. ANNs gather their knowledge by detecting the patterns and relationships in data and leaned (or rained) through experience, not from programming. There are many types of neural networks designed by now and new ones are invented every week. The behavior of a neural network is determined by transfer functions of its neurons. by learning rule, and by the architecture itself. An ANN is fonmed from artificial neuron or processing elements (PE). connected with coefficients (weights). which constitute the neural structure and are organized in layers. The first layer is termed the input layer and the last layer is the output layer. The layers of neurons between the imput and output layers are called hidden layers. The wide applicability of ANNs stems from their flexibility and ability to model nonlinear systems without prior knowledge of an empirical model. Neural networks do not need on explicit formulation of the mathematical or physical relationships of the handled problem. These give ANNs an advantage over traditional fitting methods for some chemical application. For these reason in recent years. ANNs have been used to a wide variety of chemical problems such as simulation of mass spectra. ion interaction chromatography. aqueous solubility and partition coefficient. simulation of nuclear magnetic resonance spectra. prediction of bioconcentration factor. solvent effects on reaction rate. prediction of normalized polarity parameter in mixed solvent șistems and dissociation constant of acids. $33-3$ ?

The main aim of the present work is to develop a QSPR model based on molecular descriptors using ANN for modeling and prediction of $\mathrm{E}_{\mathrm{T}} \mathrm{N}$ values for various solvents (including 216 solvents) with diverse chemical structures. In the first step, a MLR model was constructed. Then for inspection of non-linear interactions/relation between different parameters of solvents in the model, an ANN model was generated for the prediction of $\mathrm{E}_{\mathrm{T}} \mathrm{N}^{\mathrm{N}}$ values and the results were compared with the experimental and calculated values using MLR model.

\section{Theory}

A detailed description of theory behind a neural network has been adequately described by different researchers. ${ }^{19.2]}$ There are many types of neural network architectures. but the type that has been most useful for QSAR/QSPR studies is the multilayer feed - fonward network with back-propagation (BP) learning rule ${ }^{2}$ The number of neurons in the input and output layers are defined by system's properties. The number of neurons in the hidden layer could be considered as an adjustable parameter. which should be optimized. The input layer receives the experimental or theoretical information. The output layer produces the calculated values of dependent variable. The use of ANNs consists of two steps: "training" and "prediction". In the training phase the optimum structure. weight coefficients and biases are searched for. These parameters are found from a training and validation data sets. After the training phase, the trained network can be used to predict (or calculate) the outputs from a set of inputs. ANNs allow one to estimate relationships between input variables and one or several output dependent variables. Information from inputs is fed forward through the network to optimize the weights between neurons. Optimization of the weights is made by backward propagation of the error during training or learning phase. The ANN reads the input and target values in the training data set and changes the values of the weighted links to reduce the difference between the calculated output and target values. The error between output and target values is minimized across many training cycles until network reaches specified level of accuracy. If a network is left to train for too long. however. it will overtrain and will lose the ability to generalize. . $^{3+27}$

\section{Methods and Procedure}

Data set. As first step for developing the MLR and ANN models, the molecular descriptors should be generate. Normalized polarity parameter. and molecular volume of solvents are literature values. ${ }^{1,4 i}$ In order to calculate the theoretical descriptors. the z-matrices (molecular models) were constructed with the aid of HyperChem 5.01 and molecular structures were optimized using AMl algorithm. In order to calculate the theoretical descriptors and to find optimized geometries, the molecular geometries of molecules were further optimized with the same algorithm in MOPAC version 6 . The molecules in the data sets are including: alkanes. alkenes. haloalkanes. haloalkenes. cycloalkanes. 
cycloalkenes, alcohols, esters, ethers, ketones, amines, nitriles, amides, acids. phenols, hetrocyclic. nitro and aromatic compounds. The molecular descriptors were calculated for 216 solvents. The data set was randomly divided into three groups: a training set, a validation set and a prediction set consisting of 168.24 and 24 molecules, respectively. The training and validation sets were used for the model generation and the prediction set was used for the evaluation of the generated model, because a prediction set is a better estimator of the ANN generalization ability than a validation (monitoring) set."

Linear correlations. MLR model was developed for prediction of normalized polarity paraneter by molecular descriptors. The method of stepwise multi-parameter linear regression was used to select the most important descriptors and to calculate the coefficients relating the $\mathrm{E}_{i}{ }^{\wedge}$ to the descriptors. The Ml.R models were generated using spss/pc software package. Quality of the equation was indicated by the root mean square error (RMSE), Fisher index of quality $(\mathrm{F})$ and correlation coefficient $(\mathrm{R})$.

Neural network generation. The specification of a typical neural network model requires the choice of the type of inputs, the number of hidden layers, the number of neurons in each hidden layer and the connection structure between the inputs and the output layers. The number of input nodes in the ANNs was equal to the number of molecular descriptors in the MLR model. A three-layer network with a sigmoidal transfer function was designed. The initial weights were randomly selected between 0 and 1 . Before training, the input and output values were normalized between 0.1 and 0.9 . The optimization of the weights and biases was carried out according to the resilient backpropagation algorithm. ${ }^{2}$ For evaluation of the predictive power of the network, the trained ANN was used to predict $\mathrm{E}_{\mathrm{r}}{ }^{\mathrm{N}}$ values of the molecules included in the prediction set. The performances of training, validation and prediction of ANNs are evaluated by RMSE, which is defined as follows:

$$
\text { RMSE }=\sqrt{\sum_{t=1}^{N} \frac{\left(P_{t}^{\mathrm{exp}} P_{t}^{(c t)}\right)^{2}}{N}}
$$

Where $P_{j}^{\mathrm{vxp}}$ and $P_{i}^{\text {cut }}$ are experimental and calculated values of $E_{r}$ " with ANN model and $N$ denote the number of data points.

The processing of the data was carried on Intel Pentium III processor. $800 \mathrm{MHz} \mathrm{PC}$ with $256 \mathrm{Mb}$ of RAM in windows $\mathrm{XP}^{\prime}$ environment using Matlab 6.5. ${ }^{42}$ The neural networks were implemented using Neural Network Toolbox Ver. 4.0 for Matlab. ${ }^{43}$

\section{Results and Discussion}

Multi-parameter linear correlation of $\mathrm{E}_{\mathrm{T}}$ " values is. the molecular descriptors for 168 solvents in the training set gives equation (3).

$$
\mathrm{E}_{\mathrm{T}} \mathrm{Y}^{\mathrm{N}}=0.391(=0.066)+2.375( \pm 0.126) \mathrm{q}^{+}
$$

$$
\begin{aligned}
& -0.033(=0.007) \mu-0.0645( \pm 0.012) \mathrm{q} \\
& -0.115( \pm 0.024) \mathrm{V}_{\mathrm{m} 1}-2.583(=0.577) \pi_{1}
\end{aligned}
$$

$$
\left(\mathrm{n}=168, \mathrm{R}=0.874, \mathrm{RMS} \mathrm{S}=0.1043 \Gamma_{5.162}=104.92\right)
$$

$\beta_{1^{-}}=0.737, \beta_{\mu}=0.196, \beta_{\mid l}=0.245, \beta_{m 1}=-0.208, \beta \pi_{1}=-0.176$

It is clear that from cight descriptors in Table 1, rive descriptors are important in correlation of $E_{T}{ }^{\prime} w$. the molecular descriptors. As can be seen, $\Gamma_{\mathrm{T}}{ }^{\mathrm{V}}$ of solvents increase with increasing $\mathrm{q}, \mu$ and $\mathrm{q}_{\mathrm{t}}$ and decrease with $\mathrm{V}_{\mathrm{m}}$ and $\pi_{\mathrm{l}}$. Also effects of $q$ and $q$ are higher than that of the other descriptors, because standardized coefficients ( $\beta$ values) of $q^{\prime}$ and $q_{t}$ are higher than that of the other descriptors. The equation is similar to the model obtained for 30 solvents. ." With increasing $\mathrm{V}_{\mathrm{m}}$ and $\pi_{1}$ descriptors, normalized polarity parameter decrease. Because, both descriptors are indicative of dispersion effects. ${ }^{10}$ Descriptor for clectrostatic hydrogenbond acidity is q. With increasing this descriptor, the hydrogen-bonding interactions between the solvent molecules and the betaine dye increases. Dipole-dipole interactions between the molecules of solvents and betaine dye increases with increasing $\mu$ and $\mathrm{q}_{t}$ descriptors.

The next step in this work was the generation of ANN model. There are no rigorous theoretical principles for choosing the proper network topology, so different structures were tested in order to obtain the optimal hidden neurons and training cycles. ${ }^{37}$ Before training the network, the number of nodes in the hidden layer was optimized. In order to optimize the number of nodes in the hidden layer, several training sessions were conducted with different numbers of hidden nodes (from one to twelve). The root mean squared error of training (RMSITT) and validation (RMSIV) sets were obtained at various iterations for different number of neurons at the hidden layer and the minimum value of RMSEV was recorded as the optimum value. Plot of RMSET and RMSEV $v$ s. the number of nodes in the hidden layer has been shown in Figure 1. It is clear that the nine

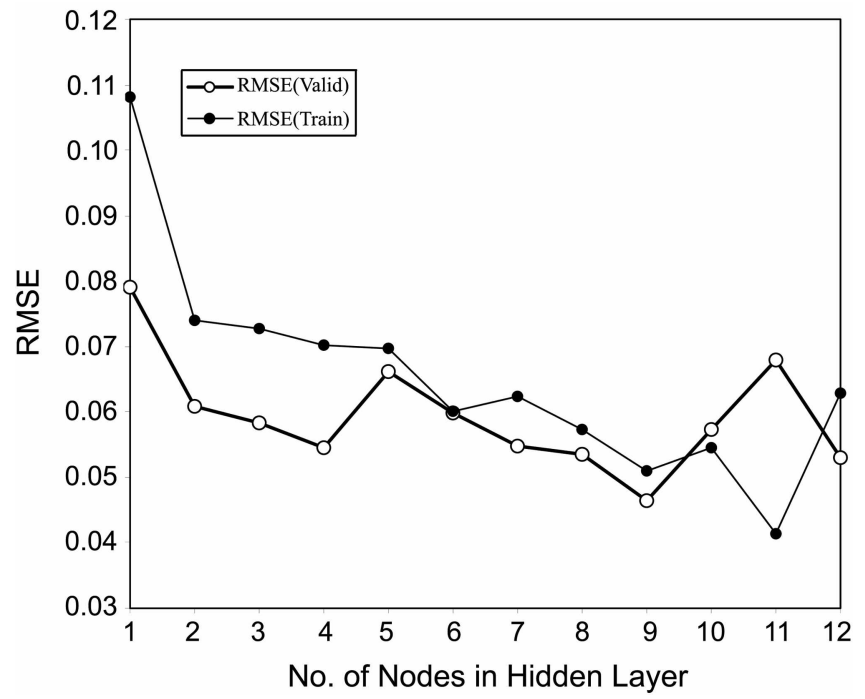

Figure 1. Plot of RMSE for training and validation sets is. the number of nodes in hidden layer. 


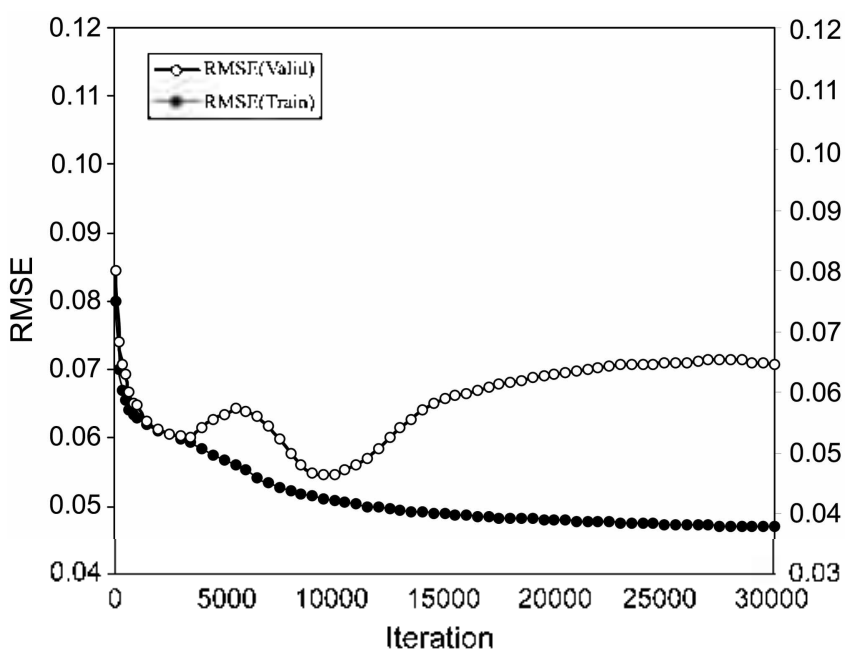

Figure 2. Plot of RMSE for calculated values of $E_{\top}{ }^{v}$ for training and validation sets $w$ s. the number of iterations.

nodes in hidden layer is optimum value.

This network consists of five inputs (including $q^{-}, \mu, \pi_{\mathrm{l}}$, $V_{m}$ and $\left.q_{l}\right)$, the same descriptors in the ML.R model, and one output for $\mathrm{E}_{\mathrm{r}}$. Then an ANN with architecture 5-9-1 was generated. It is note worthy that training of the network was stopped when the RMSEV started to increases i.e. when overtraining begins. The overtraining causes the ANN to loose its prediction power. ${ }^{37}$ Therefore, during training of the networks, it is desirable that iterations are stopped when overtraining begins. To control the overtraining of the network during the training procedure, the values of RMSE [ and RMSEV were calculated and recorded to monitor the extent of the learning in various iterations. Results obtained showed that after 10000 iterations the value of RMSEV started to increase and overfitting began (Figure 2).

The generated ANN was then trained using the training set for the optimization of the weights and biases. For the evaluation of the predictive power of the generated ANN, an optimized network was applied for prediction of the $\mathrm{E}^{\text {r. }}$ values of various solvents in the prediction set, which were not used in the modeling procedure. Then calculated values of the $\mathrm{E}_{1}$ " for various solvents in training, validation and prediction sets using the ANN model were obtained.

Figure 3 demonstrates plot of the calculated values of $\mathrm{E}_{1}{ }^{\wedge}$ for 24 solvents in prediction set versus the experimental values of it.

As expected, the calculated values of $t_{1}$ " are in good agreement with those of the experimental values. The correlation equation for the calculated values of $\mathrm{E}_{\mathrm{r}}^{\mathrm{N}}$ in prediction set using the ANN model and the experimental values is as follows:

$$
\begin{gathered}
\Gamma_{T_{T}}{ }^{\prime}(\mathrm{cal})=1.0444 \mathrm{E}_{\mathrm{T}^{\prime}}{ }^{\prime}(\exp )-0.0199 \\
\left(\mathrm{R}-0.985 ; \mathrm{RMSE}-0.0375 ; \mathrm{F}_{1.23}-741.14\right)
\end{gathered}
$$

Plot of the residual values for $\mathrm{E}_{\mathrm{r}}{ }^{\mathrm{N}}$ of solvents in prediction set versus the experimental values of it has been demonstrated in Figure 4.

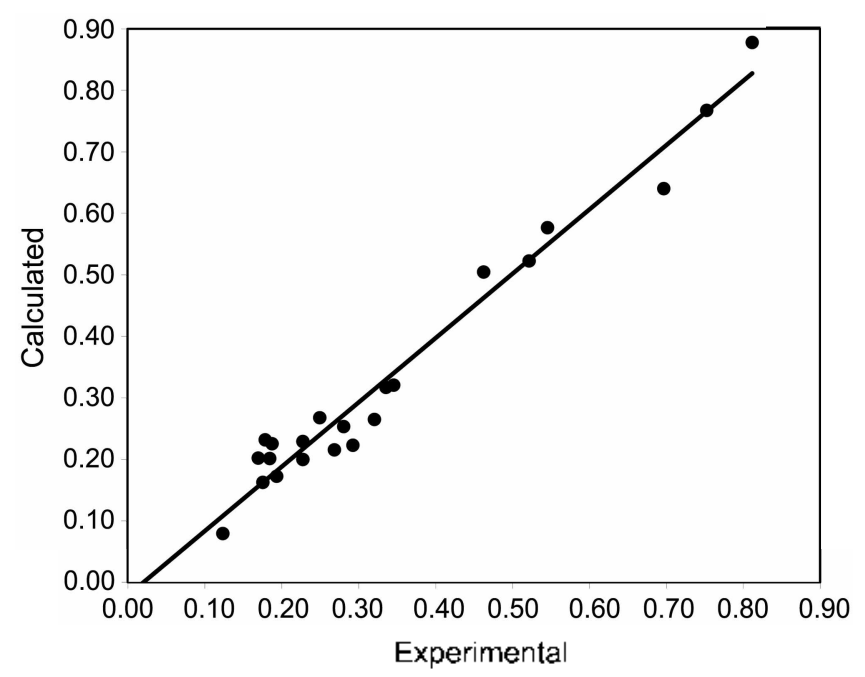

Figure 3. Plot of the calculated values of $E_{7}$ from the ANN model ws. the experimental values of it for prediction set.

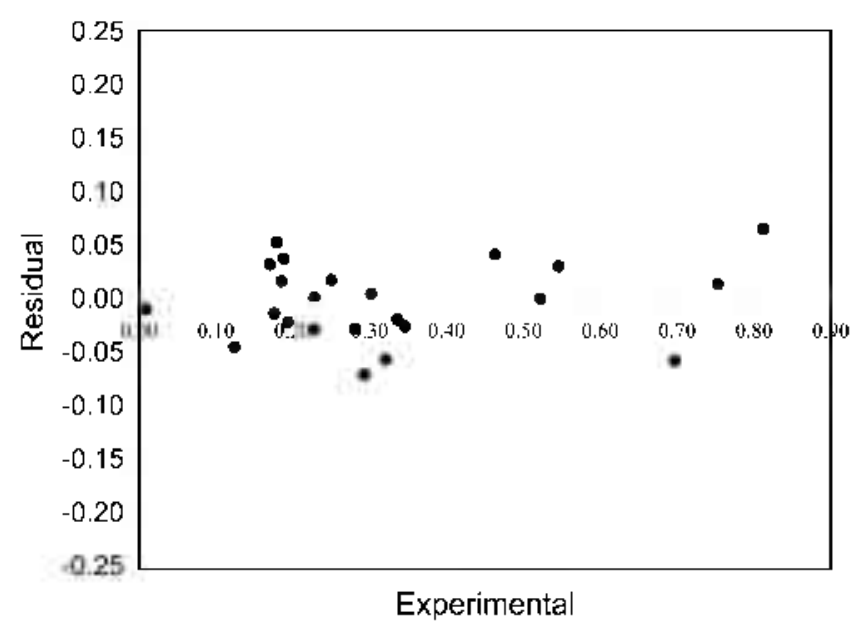

Figure 4. Plot of the Residual for calculated valus of $E^{N}{ }^{N}$ from the A. $\mathrm{N}$ model $w$ the experimental values of it for prediction set.

As can be seen the model did not show proportional and systematic error, because the slope $(a=1.0444)$ and intercept $(b=0.0199)$ of the correlation equation are not significantly different from unity and zero, respectively and the propagation of errors in both sides of zero are random shown in rigure 4.

Table 2 compares the results obtained using the MLR and ANN models. The correlation coefficient (R) and RMSE of the models for total, training, validation and prediction sets show potential of the ANN model for prediction of $E_{1}{ }^{N}$ values of various solvents.

As a result, it was found that properly selected and trained neural network could fairly represent the dependence of normalized polarity parameter on molecular descriptors. Then the optimized neural network could simulate the complicated nonlinear relationship between $\mathrm{E}_{\mathrm{r}}{ }^{\mathrm{N}}$ values and the molecular descriptors. The correlation coefficient (R) and RMSE are 0.903 and 0.0887 for the prediction set by the MLR model should be compared with the values of 0.985 
Table 2. Comparsion of statistical parameters obtained by the MLR and ANN models for correlation of nonmalized polarity parameter with molecular descriptors ${ }^{a}$

\begin{tabular}{ccccccccc}
\hline Model & $\mathrm{R}_{\text {tot }}$ & $\mathrm{R}_{\text {train }}$ & $\mathrm{R}_{\text {valia }}$ & $\mathrm{R}_{\text {rred }}$ & RMSE $_{\text {tot }}$ & RMSE $_{\text {train }}$ & RMSE $_{\text {valid }}$ & RMSE $_{\text {pred }}$ \\
\hline MLR & 0.876 & 0.874 & 0.871 & 0.903 & 0.1025 & 0.1043 & 0.1027 & 0.0887 \\
ANN & 0.973 & 0.971 & 0.975 & 0.985 & 0.0492 & 0.0510 & 0.0465 & 0.0375 \\
\hline
\end{tabular}

${ }^{a}$ tot. train. valid and pred in subseript letters are referring to the total. training. validation and prediction sets.

and 0.0375 . respectively, for the ANN model. It can be seen from Table 2 that although the paranteters appearing in the MLR model are used as inputs for the generated ANN. the statistics is shown a large iniprovement. These inprovements are due to the fact that $\mathrm{E}_{\mathrm{T}}{ }^{\mathrm{N}}$ of the solvents shows nonlinear correlations with the molecular descriptors.

\section{Conclusions}

A five-descriptor nonlinear computational neural network model has been developed for prediction of normalized polarity parameter for various solvents with diverse chemical structures using quantitative-structure property relationship. Comparison of the values of RMSE and other statistical parameters in Table 2 for training. validation and prediction sets for the models show superiority of the ANN model over the regression model. Root-mean square error of 0.0887 for the prediction set by the MLR model should be compared with the value of 0.0375 for the ANN model. Since the improvement of the results obtained using nonlinear model (ANN) is considerable. it can be concluded that the nonlinear characteristics of molecular descriptors on the $\mathrm{E}_{\mathrm{T}}{ }^{\mathrm{N}}$ values of solvents is serious and interactions between various molecular descriptors are important. Then the optimized neural network could simulate the complicated nonlinear relationship between nomalized polarity parameter and the molecular structure for various solvents

\section{References}

1. Reichardt. C. Solvents and Solvent Effects in Organic Chemish?, 3rd ed.: VCH: 2003; Chap. 4-7.

2. Marcus. Y. J. Chem. Soc. Perkin Trans. 2 1994. 1015

3. Cativiela. C.: Garcia. J. I.: Gil. J.: Martinez. R. M.: Mayoral. J. A.: Salvatella. L: Urieta. J. S.: Mainer. A. M.: Abraham. M. H. $J$. Chem. Soc.. Parkin Trans. 2 1997,653.

4. Gholami, M. R. Habibi-Yangjeh. A. Int. J. Chem. Kinet. 2000. 32,431

5. Gholami. M. R.: Habibi-Yangjeh. A. J. Phvs. Org Chent 2000. 13. 468 .

6. Gholami. M. R.: Habibi-Yangieh. A. In. J. Chem. Kintet 2001. 33, 118 .

7. Habibi-Yangjeh. A.: Gholami, M. R.: Mostaghim, R. J. Phns. Org. Chem. 2001, 14. 884.

8. Hariti. A.-R.: Habibi-Yangjeh. A.: Gholami. M. R. J. Phns. Chem. B 2006. 110.7073.

9. Todeschini. R.: Consonni. V. Handbook of Holecular Descriptors: Wiley-VCH: Weinheim, Germany. 2000.

10. Famini, G. R.: Wilson, L. Y. J. Plys. Org. Chem. 1999. 12, 645

11. Famini G. R.: Penski. C. E: Wilson. L. Y. J. Phns. Org Chem.
$1992,5,395$

12. Famini. G. R. Chemosphere 1997, 35. 2417.

13. Lowrey, A. H.: Famini, G. R.: Wilson, L. Y. J. Chem. Soc., Perkin Trans. 2 1997. 1381.

14. Cronce D. T.: Famini. G. R.: Soto. I. A. D.: Wilson. L. Y. J. Chent. Soc., Perkin Trans. 2 1998. 1293.

15. Engberts, J. B. F. N.; Famini, G. R.; Perjessy. A.: Wilson. L. Y. J. Phus. Org. Chem. 1998, 11.261.

16. Famini. G. R.: Benyamin, D; Kim. C.: Veerawat, R: Wilson, L. Y. Collect Czech. Chen Conmm 1999.64.1727.

17. Habibi-Yangjeh. A. Indicn J. Chent. B 2003. +2.1478.

18. Habibi-Yangjeh. A. Indicm J. Chent. B 2004. +3. 1504.

19. Patterson, D. W. Artificial Newal Networks: Theony and Applications, Simon and Schuster: New York. 1996; Part IП, Chap. 6.

20. Bose. N. K. Liang. P. Neural Ketwork Fudamentals: McGrawHill: New York. 1996.

21. Zupan. J.: Gasteiger. J. Nental Networks in Chentistry and Dng Design: Wiley-VCH: Weinhein. 1999.

22. Agatonovic-Kustrin, S.; Beresford. R. J. Pham Biomed. Anal. 2000. 22,717

23. Xing. W. L $;$ He X. W. Anal Chim Acta 1997. 3+9, 283.

24. Bunz. A. P.: Braun. B.: Janowshy. R. Fluid Phase Equilib. 1999. 158.367.

25. Homer. J.: Generalis. S. C.: Robson. J. H. Phvs. Chent. Chent Phus. 1999, 1,4075.

26. Goll. E. S.: Jurs. P. C. J. Chem Inf. Comp. Sci. $1999,39,974$

27. Vendrame. R.; Braga, R. S.; Takahata. Y; Galvao, D. S. I. Chem Inf. Comput Sci. 1999. 39.1094

28. Gaspelin. M.: Tusar. L.: Smid-Korbar. T.: Zupan. . T.: Kristl. T. $h$ ht. J. Phom. 2000. 196. 37.

29. Gini. G; Cracium. M. V: Konig. C.: Benfenati, E. J. Chem. Ihf. Comput. Sci. 2004, H. 1897.

30. Urata. S.: Takada, A.: Uchimaru. T.: Chandra, A. K.: Sekiva, A. $d$. Fluorine Chen. 2002. 16. 163.

31. Jalali-Heravi. M.: Masoum. S.: Shahbazikhah. P. J. Magn. Reson. 2004. 17]. 176.

32. Koziol. J. Intenet Electron. J. Mol Des. 2002. 1.80.

33. Wegner, J. K, Zell, A. J. Chem. Inf. Comput. Sci. 2003, +3, 1077

34. Valkova, I.: Vracko. M.: Basak. S. C. Anal. Chim .Acta 2004, 509. 179.

35. Jalali-Herawi. M.: Masoum. S.: Shahbazikhah. P. J. Magn. Reson. 2004. 17]. 176.

36. Habibi-Yangjeh, A; Nooshyar, M. Bull. Korean Chem. Soc. 2005. 6. 139 .

37. Habibi-Yangjeh, A.; Nooshyar. M. Phosics and Chemistry of Liquids 2005. 13. 239.

38. Habibi-Yangjeh. A.: Danandeh-Jenagharad. M.: Nooshyar. M. Bull. Korean Chent Soc. 2005. 26. 2007.

39. Habibi-Yangjeh. A.: Danandeh-Jenagharad. M.: Nooshyar. M. $d$. Mol . Model 2006. 12, 338.

40. Mareus. Y. The Properties of Solvents, John Wiley and Sons: New York. 1999.

41. Turner. J. V.: Maddalena. D. T.: Cutler. D. T. Itt. J. Phom. 2004. 270.209.

42. 1Aatlab 6.5, Mathwork: 1984-2002

43. Demuth. H. Beale, M. Neural Ketwork Toolbox: Mathworks: Natick, MA, 2000. 